\title{
Screening Study on Enzyme Inhibitory Activities of Some Mongolian Plant Leaves
}

\author{
Enkhtsetseg Enkhtuya ${ }^{1,2, *}$, Tomoko Shimamura, ${ }^{2, *}$ Takehiro Kashiwagi ${ }^{2}$, Hiroyuki Ukeda ${ }^{3}$ \\ ${ }^{1}$ School of Industrial Technology, Mongolian University of Science and Technology, Ulaanbaatar, Mongolia \\ ${ }^{2}$ Faculty of Agriculture and Marine Science, Kochi University, Kochi, Japan \\ ${ }^{3}$ Center for Regional Collaboration, Kochi University, Kochi, Japan \\ *Corresponding author: enkhtsetseg_e@must.edu.mn,tomokos@kochi-u.ac.jp
}

\begin{abstract}
In this study, 50\% ethanol extract prepared from air-dried leaves of nine plants grown wildly in Mongolia were characterized for their inhibitory activity of tyrosinase, $\alpha$-glucosidase, hyaluronidase, lipase, and angiotensin I-converting enzyme (ACE) in order to identify potential new sources for dietary prevention of certain non-communicable diseases and skin health promotion from domestic plant kingdom. The results of this study indicate that out of the selected nine Mongolian plant leaves, V.vitis-idaeae, R.altissimum, and R.acicularis leaves presented strong inhibitory activity of mushroom tyrosinase, R.acicularis and P.anomala leaves exhibited good anti $\alpha$-glucosidase and anti hyaluronidase activity, V.vitis-idaeae leaf showed appreciable inhibition of lipase activity, and P.anomala leaf effectively inhibited ACE. Among them P.anomala and R.acicularis leaves were found to be the most promising natural source with multiple biological properties.
\end{abstract}

Keywords: tyrosinase, hyaluronidase, $\alpha$-glucosidase, lipase, angiotensin I-converting enzyme, inhibitory activity, plant leaves

Cite This Article: Enkhtsetseg Enkhtuya, Tomoko Shimamura, Takehiro Kashiwagi, and Hiroyuki Ukeda, "Screening Study on Enzyme Inhibitory Activities of Some Mongolian Plant Leaves." Journal of Food and Nutrition Research, vol. 6, no. 2 (2018): 69-73. doi: 10.12691/jfnr-6-2-1.

\section{Introduction}

There are approximately 2000 edible and medical plants in Mongolia. However, scientific research into biological activities and phytochemicals of Mongolian plants is rarely carried out. In other words, many edible and medicinal plants have been largely harvested from the wild and used according to the theory of traditional folk medicine. Therefore, studies on biological activity of the plants are crucial.

According to the World Health Organization (WHO), non-communicable diseases such as cardiovascular diseases, diabetes, cancers, and chronic respiratory diseases are currently responsible for almost $70 \%$ of deaths globally. In Mongolia, the prevalence of such diseases is growing steadily, with corresponding impacts on population mortality. According to WHO surveys in Mongolia, cardiovascular diseases (hypertension, stroke and heart attack), cancer, and diabetes make $45 \%$ of total morbidity and $63 \%$ of mortality, and it was estimated that by 2020 morbidity and mortality could reach $60 \%$ and $73 \%$ respectively. Cardiovascular diseases have been the leading cause of death among Mongolians, accounting for $42 \%$ of female deaths and $35 \%$ of male deaths. Cancers are in second place, with $22 \%$ of deaths among females and $20 \%$ among males. Third place is occupied by injuries related road traffic accidents (20\% in males and $8 \%$ in females). Of all non-communicable diseases, diabetes accounts for $1 \%$, which is 49.9 per 10000 population. Women are affected more compared to men. Key risk factors of these noncommunicable diseases are smoking, harmful patterns of alcohol use, unhealthy diets with too much fat and salt, insufficient fruits and vegetables, physical inactivity, overweight and obesity. To prevent and control such diseases, reduction of risky behaviors, early detection and developing functional foods with multiple biological properties by using non-traditional food resources are necessary.

In our continuing search for alternative bioactive agents from domestic plant kingdom, leaves of following wild plants grown in Mongolia were investigated: Ribes nigrum, Crataegus sanguinea, Vaccinium vitis-idaeae, Paeonia anomala, Rosa acicularis, Hippophae rhamnoides, Fragaria orientalis, Ribes diacanthum and Ribes altissimum. The selected plants belong to different families such as Paeoniaceae (P.anomala), Elaeagnaceae (H.rhamnoides), Ericaceae (V.vitis-idaeae), Grossulariaceae (R.nigrum, R.altissimum, and R.diacanthum) and Rosaceae (R.acicularis, C.sanguinea, and F.orientalis). In our previous study, the antioxidant potential of these plant leaves were evaluated in vitro with five different analytical methods including DPPH radical scavenging assay, ABTS radical cation scavenging assay, superoxide anion scavenging assay, ferric reducing antioxidant power assay, and ferrous ions chelation assay. Moreover, contents of polyphenolics, namely total phenolics, total flavonoids, and total flavanols, were also estimated by biochemical methods. Among the selected plant leaves, V.vitis-idaeae, P.anomala, R.acicularis, 
and H.rhamnoides had significant antioxidant potential and were rich in polyphenolics. In particular, P.anomala leaf showed remarkable scavenging activity against DPPH radicals, ABTS radical cations and superoxide anions, and exerted significant ferric reducing antioxidant power [1]. Afterwards, we identified three main constituents in P.anomala leaf, namely methyl gallate, pentagalloylglucose, and tellimoside, which showed potent DPPH radical scavenging activity [2]. Antioxidant activity is a fundamental property important for life. Many of the biological functions, such as antimutagenicity, anticarcinogenicity, and antiaging, among others, originate from this property. Many of the natural antioxidants, especially flavonoids, exhibit a wide range of biological effects, including antibacterial, antiviral, anti-inflammatory, antiallergic, antithrombotic, and vasodilatory actions [3]. The present study was therefore designed to evaluate effects of the selected nine plant leaves on tyrosinase, hyaluronidase, $\alpha$-glucosidase, lipase, and angiotensin I-converting enzyme (ACE) activity in order to identify potential new sources for dietary prevention of leading non-communicable diseases in Mongolia and skin health promotion. The selected plant leaves are described for the first time for those biological properties. To our best knowledge, there are no scientific studies on the chemistry and beneficial biological functions such as the enzyme inhibitory activities of C.sanguinea, P.anomala, R.acicularis, R.diacanthum and R.altissimum leaves. The previous phytochemical investigations of F.orientalis, R.nigrum, H.rhamnoides, and V.vitis-idaeae did not reveal the presence of natural compounds with the enzyme inhibitory effects.

\section{Materials and Methods}

\subsection{Chemicals}

L-DOPA (3,4-dihydroxy-L-phenyl-alanine), mushroom tyrosinase (EC 1.4.18.1., activity $~ 5771$ units/mg of solid), bovine testicular hyaluronidase (EC 3.2.1.35., activity $750-3000$ units/mg of solid), compound 48/80 (condensation product of $N$-methyl-p-methoxyphenethylamine with formaldehyde), hyaluronic acid sodium salt from rooster comb, intestinal acetone powders from rat were purchased from Sigma-Aldrich (St. Louis, MO., USA). Glucose C-II test kit, Lipase Kit S, and ACE kit-WST were obtained from Wako Pure Chemicals (Osaka, Japan), DS Pharma Biomedical Co., Ltd (Osaka, Japan), and Dojindo Laboratories (Kumamoto, Japan), respectively. All other chemicals used for analyses were analytical reagent grade and were used without further purification. All solutions were prepared with water purified by Auto Pure WQ501 system (Yamato Scientific Co., Ltd, Tokyo, Japan).

\subsection{Plant Materials}

Fresh leaves of the selected plants were collected from Tuv province of Mongolia in the beginning of August, 2015. These plants were identified in the Institute of Biology, Mongolian Academy of Science. Well-grown and healthy leaves were collected without its petiole, air-dried at room temperature in the shade to constant weights and powdered.

\subsection{Sample Extraction}

Two grams of each sample were extracted with $40 \mathrm{~mL}$ of $50 \%(\mathrm{v} / \mathrm{v})$ ethanol in water on a magnetic stirrer for $2 \mathrm{~h}$ at room temperature and centrifuged at $3000 \times g$ for 10 $\min$ at $4^{\circ} \mathrm{C}$. The supernatant was filtered with No.5C paper (Advantec Toyo, Tokyo, Japan) and decanted into $100 \mathrm{~mL}$ volumetric flask. The pellet was extracted again under identical condition. The supernatants were combined, diluted to $100 \mathrm{~mL}$ with $50 \%$ ethanol and stored at $-20^{\circ} \mathrm{C}$ until use.

\subsection{Determination of Enzyme Inhibitory Activity}

\subsubsection{Determination of Tyrosinase Inhibitory Activity}

The enzymatic assay for tyrosinase inhibitory activity was determined as described by Kubo and Kinst-Hori with modifications (1998). Tyrosinase solution was prepared using mushroom tyrosinase dissolved in $50 \mathrm{mM}$ potassium phosphate buffer ( $\mathrm{pH}$ 6.5) to give 71.5 units $/ \mathrm{mL}$, which was stored $-20^{\circ} \mathrm{C}$ until use. In a 96-well microplate, 140 $\mu \mathrm{L}$ of $0.1 \mathrm{M}$ sodium phosphate buffer ( $\mathrm{pH} 6.8), 4 \mu \mathrm{L}$ of sample solution, and $60 \mu \mathrm{L}$ of the tyrosinase solution were added, followed by 5 min of pre-incubation at $25^{\circ} \mathrm{C}$ on a shaker. Then $60 \mu \mathrm{L}$ of $5 \mathrm{mM}$ L-DOPA was added to each well. The mixture was incubated for exactly $5 \mathrm{~min}$ at $25^{\circ} \mathrm{C}$ on the shaker and measured at the absorbance of $475 \mathrm{~nm}$ by using a microplate reader (Sunrise Rainbow RC; Tecan Group Ltd., Mannedorf, Switzerland). The 50\% ethanol was used as a control. Blank solutions with and without enzyme were also prepared with no sample solution added. The inhibition rate was calculated using the following equation:

$$
\text { Inhibition rate }(\%)=\left[1-\frac{\left(A_{S}-A_{S b}\right)}{\left(A_{C}-A_{C b}\right)}\right] \times 100
$$

where $A_{S}$ is the absorbance of sample with tyrosinase, $A_{S b}$ is the absorbance of sample without tyrosinase, $A_{C}$ is the absorbance of control with tyrosinase and $\mathrm{A}_{\mathrm{Cb}}$ is the absorbance of control without tyrosinase.

\subsubsection{Determination of Hyaluronidase Inhibitory Activity}

Hyaluronidase inhibitory activity of the selected plant leaves was evaluated according to the method Kakegawa et al. and Maeda et al. with some modifications. Before the enzymatic assay, 3200 units/mL of hyaluronidase stock solution was prepared with water and stored at $-20^{\circ} \mathrm{C}$ until use. Hyaluronidase solution was defrosted and diluted twice with $0.1 \mathrm{M}$ acetate buffer ( $\mathrm{pH} \mathrm{4.0)}$ when used. The activating solution was prepared by adding $1 \mathrm{mg}$ of compound $48 / 80$ and $7.5 \mathrm{mg}$ of calcium chloride dihydrate into $10 \mathrm{~mL}$ of $0.1 \mathrm{M}$ acetate buffer ( $\mathrm{pH} 4.0)$. Potassium borate solution was prepared by adding $2.24 \mathrm{~g}$ of potassium hydroxide to $1 \mathrm{~L}$ of $0.8 \mathrm{M}$ boric acid. $p$-Dimethylaminobenzaldehyde (DMAB) solution, which is color development reagent, was prepared dissolving $100 \mathrm{mg}$ of DMAB in $1 \mathrm{~mL}$ of the mixed solution containing $6 \mathrm{~mL}$ of $10 \mathrm{~N}$ hydrochloric acid and $44 \mathrm{~mL}$ of acetic acid. DMAB solution was diluted ten times with acetic acid before use. 
In a sample tube, $30 \mu \mathrm{L}$ of sample and $15 \mu \mathrm{L}$ of 1600 units/mL hyaluronidase solution were added and incubated for $20 \mathrm{~min}$ at $37^{\circ} \mathrm{C}$, followed by addition of 30 $\mu \mathrm{L}$ of the activating solution. The sample mixture was further incubated for $20 \mathrm{~min}$ at $37^{\circ} \mathrm{C}$. After incubation, 75 $\mu \mathrm{L}$ of $0.32 \mathrm{mg} / \mathrm{ml}$ of sodium hyaluronate in acetate buffer was added to start the reaction. The sample mixture was incubated for exactly $60 \mathrm{~min}$ at $37^{\circ} \mathrm{C}$, and $30 \mu \mathrm{L}$ of $0.4 \mathrm{~N}$ sodium hydroxide was added to stop the reaction. The inhibition on bovine testicular hyaluronidase was tested by Morgan-Elson method with some modifications in the procedure [5]. Briefly, the sample mixture, to which 30 $\mu \mathrm{L}$ of the potassium borate solution was added, was heated at $100^{\circ} \mathrm{C}$ for exactly $3 \mathrm{~min}$. The sample was cooled immediately in cold water bath. In 96-well microplate, 84 $\mu \mathrm{L}$ of the sample mixture and $180 \mu \mathrm{L}$ of DMAB solution were added and incubated at $37^{\circ} \mathrm{C}$ for exactly $20 \mathrm{~min}$. Then, absorbance of the sample solution was measured at $585 \mathrm{~nm}$. The initial crude extracts of the selected plant leaves were diluted with $50 \%$ ethanol to 1,5 , and $10 \mathrm{mg}$ dry leaf $/ \mathrm{mL}$, and the experiments were triplicated. The following equation was used to determine the inhibitory activity of the sample:

$$
\text { Inhibition rate }(\%)=\left[1-\frac{\left(A_{S}-A_{S b}\right)}{\left(A_{C}-A_{C b}\right)}\right] \times 100
$$

where $A_{S}$ is the absorbance of sample with hyaluronidase, $\mathrm{A}_{\mathrm{Sb}}$ is the absorbance of sample without hyaluronidase, $\mathrm{A}_{\mathrm{C}}$ is the absorbance of control with hyaluronidase and $\mathrm{A}_{\mathrm{Cb}}$ is the absorbance of control without hyaluronidase.

\subsubsection{Determination of $\alpha$-Glucosidase Inhibitory Activity}

Rat intestinal acetone powders were used as a source of enzyme $\alpha$-glucosidase. For extraction of the $\alpha$-glucosidase, $0.1 \mathrm{~g}$ of rat intestinal acetone powder were homogenized with $1 \mathrm{~mL}$ of $0.1 \mathrm{M}$ phosphate buffer at $\mathrm{pH} 7.0$ for $1 \mathrm{~h}$ on crushed ice and centrifuged at $6000 \times \mathrm{g}$ for $45 \mathrm{~min}$ at $4^{\circ} \mathrm{C}$. The supernatant obtained before the experiment was used the enzyme solution for the $\alpha$-glucosidase reaction and saved in crushed ice. The enzyme solution was diluted 10 times by phosphate buffer ( $\mathrm{pH} 7.0) 5$ min prior to use. In 96-deep well plate, $25 \mu \mathrm{L}$ of sample solution was mixed with $100 \mu \mathrm{L}$ of $0.1 \mathrm{M}$ phosphate buffer ( $\mathrm{pH} 7.0)$ and 100 $\mu \mathrm{L}$ of $0.25 \mathrm{M}$ maltose in phosphate buffer at $\mathrm{pH} 7.0$, and the mixture was pre-incubated for $5 \mathrm{~min}$ at $37^{\circ} \mathrm{C}$. The reaction was started by adding $25 \mu \mathrm{L}$ of $\alpha$-glucosidase from rat intestinal acetone powder to the sample mixture. The enzymatic reaction was allowed to proceed at $37^{\circ} \mathrm{C}$ for $40 \mathrm{~min}$, and then stopped by adding $250 \mu \mathrm{L}$ of $0.2 \mathrm{M}$ sodium carbonate. The glucose released in the reaction mixture was determined by the Glucose C-II test kit at $505 \mathrm{~nm}$. A control was run with $25 \mu \mathrm{L}$ of distilled water instead of the sample extract. For blank determination (to allow for absorbance produced by the extract solution), the enzyme solution was replaced with $0.1 \mathrm{M}$ phosphate buffer at $\mathrm{pH} 7.0$ and the same procedure was carried out as above.

The percent inhibition of $\alpha$-glucosidase activity was calculated as follows:

$$
\% \text { inhibition }=\left(\mathrm{A}_{\mathrm{C}}-\mathrm{A}_{\mathrm{S}}\right) / \mathrm{A}_{\mathrm{C}} \times 100
$$

where $A_{S}$ is the difference in absorbance decrease at 505 $\mathrm{nm}$ between blank and the sample; $\mathrm{A}_{\mathrm{C}}$ is the absorbance of the control.

\subsubsection{Determination of Lipase Inhibitory Activity}

Lipase inhibitory activity was evaluated according to the instruction of Lipase Kit S.

\subsubsection{Determination of Angiotensin I-Converting Enzyme Inhibitory Activity}

ACE inhibition activity of the selected plant leaves was evaluated by ACE kit-WST. The experiments and calculations to evaluate ACE inhibitory activity of selected plant leaves were performed according to manufacturer's instructions.

\subsection{Statistical Analyses}

All results are expressed as mean \pm standard deviation (SD) of at least three determinations. The $\mathrm{IC}_{50}$ values, the concentrations producing $50 \%$ inhibitory activity, were calculated by interpolation of concentration-inhibition curves. Microsoft Excel software was used for the calculation.

\section{Results and Discussion}

We have screened nine plant leaves grown in Mongolia for their inhibitory activities of tyrosinase, hyaluronidase, $\alpha$-glucosidase, lipase, and ACE. This study is based on spectrophotometric assay. The enzyme inhibitory activities were expressed as percentage of inhibition. The $\mathrm{IC}_{50}$ values were determined for some plant leaves which showed more than $50 \%$ inhibition of enzyme activity at the initial concentration of the undiluted sample extracts. All $\mathrm{IC}_{50}$ values were calculated from the corresponding dose inhibition curve.

The aqueous extracts of the plant contain alkaloids, flavonoids, oils, phenolic compounds and tannins and the alcoholic extracts contain alkaloids, carbohydrates, flavonoids, proteins, cardiac glycosides, oils, phenolic compounds and tannins. The phytochemical analysis indicated that ethanol can extract more active principles of the plant than the water [8]. Therefore, ethanol is frequently used in food and drug industry. In this study, $50 \%$ ethanol in water was chosen as extraction solvent. The $50 \%$ ethanol in water has moderate polarity and able to extract both hydrophilic and hydrophobic bioactive compounds.

Table 1 summarizes results of mushroom tyrosinase, $\alpha$-glucosidase, ACE, lipase, and bovine testicular hyaluronidase inhibitions of the selected nine plant leaves. In this table, values are presented as percentage (\%) of enzyme inhibitions.

\subsection{Tyrosinase Inhibitory Activity}

The tyrosinase inhibitory activity of the plant leaves was examined in vitro by monitoring the appearance of dopachrome, an intermediate in the melanogenesis process. L-DOPA was used as the substrate for mushroom 
tyrosinase. As shown in Table 1, all tested plant leaves showed more than $30 \%$ inhibition of tyrosinase activity at the concentration of $20 \mathrm{mg} / \mathrm{mL}$. Among them, five plant leaves exerted more than $50 \%$ inhibition. Out of these five plant leaves, V.vitis-idaeae, R.acicularis, and R.altissimum present relatively strong inhibition of mushroom tyrosinase with values around $75 \%$. V.vitis-idaeae showed the greater percentage inhibition of the tyrosinase enzyme compared to other plant leaves. The plant leaf extracts V.vitis-idaeae, R.altissimum, R.acicularis, P.anomala, and R.nigrum showed an $\mathrm{IC}_{50}$ value of $4.15 \pm 0.19,8.37 \pm 0.58$, $9.96 \pm 0.40,13.29 \pm 0.76$, and $14.36 \pm 0.93 \mathrm{mg} / \mathrm{mL}$ respectively in the tyrosinase inhibition assay. There was a dose dependent increase in percentage inhibitory activity against tyrosinase by all the five plant leaves. It is known that arbutin and hydroquinone, which are well-known skin whitening and depigmentation agents $[9,10]$, naturally occur in air-dried leaves of V.vitis-idaeae. Therefore, arbutin and hydroquinone may be responsible for its good tyrosinase inhibitory potency.

Wardhan et al. compared mushroom tyrosinase inhibitory activity of 30 Indian plant extracts including V.vitis-idaeae fruit and F.ananassa fruit which is cultivated strawberry in a garden. Ethyl acetate extract of $F$.ananassa fruit was one of the most active plant extracts against mushroom tyrosinase, whereas hexane, ethyl acetate, methanol and water extracts of $V$.vitis-idaeae fruit showed relatively weak inhibition of tyrosinase. The other extracts of F.ananassa fruit exerted also relatively weak tyrosinase inhibitory activity.

Table 1. Enzyme Inhibiting Potentials of the Selected Plant Leaves

\begin{tabular}{|c|c|c|c|c|c|}
\hline \multirow{2}{*}{ Plant leaf } & $\begin{array}{c}\text { Tyrosinase inhibition } \\
(\%)\end{array}$ & $\begin{array}{l}\text { Hyaluronidase } \\
\text { inhibition }(\%)\end{array}$ & $\begin{array}{c}\alpha \text {-Glucosidase inhibition } \\
(\%)\end{array}$ & $\begin{array}{c}\text { Lipase inhibition } \\
(\%)\end{array}$ & ACE inhibition (\%) \\
\hline & $20 \mathrm{mg} / \mathrm{mL}$ & $5 \mathrm{mg} / \mathrm{mL}$ & $20 \mathrm{mg} / \mathrm{mL}$ & $20 \mathrm{mg} / \mathrm{mL}$ & $0.4 \mathrm{mg} / \mathrm{mL}$ \\
\hline R.nigrum & $66.36 \pm 3.21$ & $87.31 \pm 3.80$ & $9.29 \pm 1.40$ & $10.34 \pm 1.10$ & $47.73 \pm 1.38$ \\
\hline C.sanguinea & $31.25 \pm 1.94$ & $33.29 \pm 1.69$ & $-7.81 \pm 0.50$ & $3.48 \pm 0.10$ & $7.43 \pm 0.17$ \\
\hline V.vitis-idaeae & $76.59 \pm 2.12$ & $109.11 \pm 0.30$ & $4.64 \pm 0.40$ & $58.77 \pm 5.12$ & $19.71 \pm 0.78$ \\
\hline P.anomala & $56.97 \pm 2.76$ & $86.09 \pm 0.97$ & $50.56 \pm 1.89$ & $17.19 \pm 0.80$ & $59.38 \pm 1.97$ \\
\hline R.acicularis & $74.39 \pm 2.73$ & $106.81 \pm 0.74$ & $61.34 \pm 2.29$ & $43.43 \pm 2.91$ & $21.70 \pm 1.08$ \\
\hline H.rhamnoides & $38.54 \pm 2.26$ & $48.10 \pm 5.86$ & $18.59 \pm 1.23$ & $28.55 \pm 1.85$ & $8.15 \pm 0.40$ \\
\hline F.orientalis & $31.42 \pm 3.01$ & $45.13 \pm 3.77$ & $11.34 \pm 0.56$ & $13.96 \pm 1.13$ & $-0.38 \pm 0.01$ \\
\hline R.altissimum & $75.26 \pm 1.91$ & $105.98 \pm 1.25$ & $10.78 \pm 0.91$ & $44.96 \pm 3.52$ & $32.45 \pm 1.52$ \\
\hline R.diacanthum & $37.60 \pm 1.73$ & $75.03 \pm 1.91$ & $20.40 \pm 1.22$ & - & $10.7 \pm 0.43$ \\
\hline
\end{tabular}

- not determined because of sample lack.

\subsection{Hyaluroniadase Inhibitory Activity}

The hyaluronidase inhibitory activity of the selected plant leaves was ascertained spectrophotometrically in vitro at a concentration of $5 \mathrm{mg} / \mathrm{mL}$ by using hyaluronic acid from rooster comb and bovine testicular hyaluronidase. The hyaluronidase inhibitory activity was in the following decreasing order: V.vitis-idaeae $>$ R.acicularis $>$ R.altissimum $>$ R.nigrum $>$ P.anomala $>$ R.diacanthum $>$ H.rhamnoides $>$ F.orientalis $>$ C.sanguinea. The hyaluronidase $\mathrm{IC}_{50}$ values for R.acicularis, P.anomala, V.vitis-idaeae, R.altissimum, and R.nigrum were determined as $0.88 \pm 0.06$, $1.07 \pm 0.06,2.21 \pm 0.08,2.66 \pm 0.11$, and $3.08 \pm 0.12 \mathrm{mg} / \mathrm{mL}$ respectively. The percent inhibition of hyaluronidase activity was found to be increased as concentration of the extracts increased. As V.vitis-idaeae leaf demonstrated maximum hyaluronidase inhibitory activity $(109.11 \pm 0.30 \%)$ at a concentration of $5 \mathrm{mg} / \mathrm{mL}$, its $\mathrm{IC}_{50}$ value was more than those of P.anomala and R.acicularis. Similarly, R.altissimum was more effective than P.anomala at 5 $\mathrm{mg} / \mathrm{mL}$; however its $\mathrm{IC}_{50}$ value was more than those of P.anomala. It means that the inhibition effect of V.vitisidaeae and R.altissimum drastically dropped at lower concentration. According to the percent inhibition and $\mathrm{IC}_{50}$ value, P.anomala leaf was less effective than R.acicularis. Therefore, R.acicularis leaf was ranked first out of tested nine plant leaves by hyaluronidase inhibitory potencial followed by P.anomala, V.vitis-idaeae, R.altissimum and R.nigrum. Since H.rhamnoides, F.orientalis, and C.sanguinea showed less than $50 \%$ inhibition of hyaluronidase activity at the concentration of $5 \mathrm{mg} / \mathrm{mL}, \mathrm{IC}_{50}$ values for those plants were not determined. The hyaluronidase inhibiting potency of C.sanguinea was the minimum when compared to the other plant extract.

\section{3. $\alpha$-Glucosidase Inhibitory Activity}

Except for C.sanguinea, all tested plant leaves inhibited $\alpha$-glucosidase at a concentration of $20 \mathrm{mg} / \mathrm{mL}$. This study revealed that six from nine plant leaves present a poor $\alpha$ glucosidase inhibitory activity (less than 20\%). While at the same condition, R.acicularis and P.anomala exerted the greater percent inhibition of the $\alpha$-glucosidase enzyme compared to other plant leaves. The $\alpha$-glucosidase $\mathrm{IC}_{50}$ values for R.acicularis and P.anomala were determined as $16.43 \pm 0.89$ and $19.79 \pm 1.25 \mathrm{mg} / \mathrm{mL}$, respectively. Therefore, R.acicularis and P.anomala could be useful in management of postprandial hyperglycemia and diabetes prevention.

\subsection{Lipase Inhibitory Activity}

Of the nine plant leaves evaluated, the highest inhibitory activity against lipase was exerted by V.vitisidaeae with $\mathrm{IC}_{50}$ value of $17.66 \pm 1.12 \mathrm{mg} / \mathrm{mL}$. Next, R.altissimum and R.acicularis showed possible inhibitory activity on lipase. At a concentration of $20 \mathrm{mg} / \mathrm{mL}$, the 
inhibition effects of R.altissimum and R.acicularis were $44.96 \pm 3.52$ and $43.43 \pm 2.91 \%$, respectively. The other plant leaves produced relatively weak lipase inhibition (less than 30\%). Interestingly, P.anomala, which possessed remarkable anti-tyrosinase, anti-hyaluronidase, anti-glucosidase, and ACE inhibiting potential, could not effectively inhibit lipase. Its inhibition effect was found to be just $17.19 \pm 0.80 \%$ at $20 \mathrm{mg} / \mathrm{mL}$.

\subsection{ACE Inhibitory Activity}

The ACE kit-WST is used for the determination of ACE inhibitory activity of the selected plant leaves. When use this kit, final concentration of organic solvents such as dimethyl sulfoxide (DMSO) and ethanol should be lower than $1 \%$. Therefore, the initial ethanol extract of the selected plant leaves was diluted with distilled water 50 times to give $0.4 \mathrm{mg}$ dry leaf $/ \mathrm{mL}$. Among the tested plant leaves, only F.orientalis leaf showed no inhibition of ACE activity at $0.4 \mathrm{mg} / \mathrm{mL}$ concentration. Under the same condition, P.anomala leaf showed highest ACE inhibitory activity with $\mathrm{IC}_{50}$ value of $0.35 \pm 0.01 \mathrm{mg} / \mathrm{mL}$. However, R.nigrum (47.73\%), R.altissimum (32.45\%), and R.acicularis (21.70\%) exhibited reasonable ACE inhibitory activity. Relatively weak potential (less than $20 \%$ ) to inhibit ACE was observed in the remaining four plant leaves. This result indicated that P.anomala leaf could be useful in the hypertension management.

The results of this study indicate that out of the tested nine Mongolian plant leaves, V.vitis-idaeae, R.altissimum, and R.acicularis leaves presented strong inhibitory activity of mushroom tyrosinase, R.acicularis and P.anomala leaves exhibited good anti $\alpha$-glucosidase and anti hyaluronidase activity, V.vitis-idaeae leaf showed appreciable inhibition of lipase activity, and P.anomala leaf effectively inhibited ACE. Therefore, P.anomala leaf might be new potential source of blood-glucose lowering, skin-lightening, anti-wrinkle, anti-inflammatory and antihypertensive agents suitable for use as cosmetics and functional foods and beverages in future. V.vitis-idaeae leaf could represent possible novel source of skin-whitening and anti-obesity agent. In case of R.acicularis leaf, it could be new powerful anti-diabetic, anti-wrinkle, and anti-inflammatory material.

To our best knowledge, there are no scientific studies on the phytochemistry and beneficial biological functions such as the enzyme inhibitory activities of C.sanguinea, P.anomala, R.acicularis, R.diacanthum and R.altissimum leaves. The previous phytochemical investigations of F.orientalis, R.nigrum, H.rhamnoides, and V.vitis-idaeae did not reveal the presence of natural compounds with the enzyme inhibitory effects. In previous study we identified three major antioxidative constituents in P.anomala leaf, namely methyl gallate, pentagalloylglucose, and quercetingalloylglucose. These three compounds contributed more than $30 \%$ of the total DPPH radical scavenging activity of the initial extract [2]. Since many biological functions of plants originate from antioxidant property, the identified three antioxidative compounds in P.anomala leaf may influence on its multiple biological properties.

\section{Conclusion}

Among the tested nine plant leaves, P.anomala and R.acicularis leaves were found to be the most promising natural source with multiple biological properties including anti-diabetic, anti-melanogenesis, anti-wrinkle, anti-inflammatory and antihypertensive. However, C.sanguinea, F.orientalis, R.diacanthum, and H.rhamnoides showed the least inhibitory activities in the all used assays. Further investigations will focus on the isolation and chemical identification of the major principles from the most active plant leaves.

\section{Acknowledgments}

This work was financially supported by JSPS (Japan Society for the Promotion of Science) RONPAKU (Dissertation PhD) Program (113209).

\section{Statement of Competing Interests}

The authors have no competing interests.

\section{References}

[1] Enkhtuya E, Kashiwagi T, Shimamura T, Ukeda H, Tseye-Oidov $\mathrm{O}$, "Screening study on antioxidant activity of plants grown wildly in Mongolia", Food Science and Technology Research, 20 (4). 891-897. 2014.

[2] Enkhtuya E, Kashiwagi T, Shimamura T, Ukeda H, "Antioxidative constituents in the leaves of Paeonia anomala grown in Mongolia", Food Science and Technology Research, 23 (1). 63-70. 2017.

[3] Cook N.C, Samman S, "Flavonoids-Chemistry, metabolism, cardioprotective effects, and dietary sources", Journal of Nutritional Biochemistry, 7. 66-76. 1996.

[4] Kubo I, Kinst-Hori I, "Tyrosinase Inhibitors from Anise Oil", Journal of Agricultural and Food Chemistry, 46. 1268-1271. 1998.

[5] Kakegawa H, Matsumoto H, Satoh T, "Activation of hyaluronidase by metallic salts and compound $48 / 80$, and inhibitory effect of anti-allergic agents on hyaluronidase", Chemical and Pharmaceutical Bulletin, 33. 642-646. 1985.

[6] Maeda Y, Yamamoto M, Matsui T, Sugiyama K, Yokota M, Nakagomi K, Tanaka H, Takahashi I, Kobayashi T, Kobayashi E, "Inhibitory effect of tea extracts on hyaluronidase (Studies on antiallergic activity in tea. II)", Journal of the Food Hygienic Society of Japan, 31. 233-237. 1990.

[7] http://www.dojindo.com/store/p/197-ACE-Kit-WST.html

[8] Asha Gangadharan, Elizabeth Jacob and Densely Jose, "Phytochemical analysis, antibacterial and anti-hyaluronidase avtivity of three indigenous medicinal plants", World Journal of Pharmacy and Pharmaceutical Sciences, 3 (6). 751-761. 2014.

[9] S.Momtaz, N.Lall and A.Basson. "Inhibitory activities of mushroom tyrosine and DOPA oxidation by plant extracts", South African Journal of Botany, 74. 577-582. 2008.

[10] K.T.Lee, B.J.Kim, J.H.Kim, M.Y.Heo and H.P.Kim. "Biological screening of 100 plant extracts for cosmetic use (I): inhibitory acivities of tyrosinase and DOPA auto-oxidation", International Journal of Cosmetic Science, 19. 291-298. 1997

[11] Anand Wardhan, Shabina Khan and Bhawana Pandey. "Screening of plant parts for anti-tyrosinase activity by tyrosinase assay using mushroom tyrosinase", Indian Journal of Scientific Research, 4 (1). 134-139. 2014. 